\title{
Monolithic dynamic covalent polymer aerogels via ambient pressure drying with super-flexibility, weldability, closed-loop recyclability, and multifunctionality
}

\author{
Xinhai Zhang ${ }^{1}$, Jun Zhao ${ }^{1}$, Kai Liu ${ }^{1}$, Guangfeng Li ${ }^{1}$, Dong Zhao ${ }^{1}$, Zhaoming Zhang ${ }^{1}$, Junjun Wan ${ }^{1}$, \\ Xue Yang ${ }^{1}$, Ruixue Bai ${ }^{1}$, Yongming Wang ${ }^{1}$, Wei Zhang ${ }^{2 *}$, and Xuzhou Yan ${ }^{1 *}$ \\ ${ }^{1}$ School of Chemistry and Chemical Engineering, Frontiers Science Center for \\ Transformative Molecules, Shanghai Jiao Tong University, Shanghai 200240, P. R. China \\ ${ }^{2}$ Department of Chemistry, University of Colorado, Boulder, CO 80309, USA \\ *Corresponding authors. E-mail: xzyan@sjtu.edu.cn; wei.zhang@colorado.edu
}

\begin{abstract}
Owing to their low density, high porosity and unique micro-nanostructures, aerogels are attractive for applications in various aspects, but at the same time, suffer from shrinkage and/or cracking during preparation, mechanical brittleness, low production efficiency, and non-degradation. Herein, we introduce the concept of dynamic covalent polymer chemistry to produce a new class of aerogels - referred to as DCPAs. The resulting light DCPAs display large-scale preparation prospect and feature high porosity (90.7-91.3\%), large degrees of compression (80\% strain) and bending (diametral deflection of $30 \mathrm{~mm}$ ) without any cracks, as well as considerable tensile property (elongation at break of 32.7\%). In addition, our DCPAs showcase emergent characteristics of weldability, repairability, and closed-loop recyclability that are highly desirable for affording versatile materials platforms but hardly achieved by traditional aerogels. Benefiting from the robust porous structures, we demonstrate the potential of the DCPAs for applications in thermal insulation and emulsion separation. These findings reveal that the dynamic covalent bond strategy would be generalized for the production of a new generation of aerogels with customized features for functioning in the field of intelligent and sustainable materials.
\end{abstract}

\section{Introduction}

Weight reduction, performance improvement, and cost reduction have been the most significant drives for the design of structural materials (1). Aerogels, which are porous sol-gel materials, have been recognized as pivotal components for advanced structural materials due to their fascinating characteristics of low density $\left(0.001-0.3 \mathrm{~g} / \mathrm{cm}^{3}\right)$ and high porosity $(>80 \%)(2-4)$. They have been widely utilized in various fields, such as energy storage (5), drug delivery (6), sensors (7), sound absorption (8), electromagnetic shielding (9), thermal insulation (4), water treatment (10), and so on. However, inevitable volume shrinkage and/or cracking induced by capillary force during the drying process together with brittle nature of aerogels themselves have been the long-standing and intractable problems (11). To fabricate monolithic aerogels with desired performances, special drying methods that can decrease or eliminate the undesirable capillary force, such as supercritical drying and freeze drying, are always considered, but low production and high cost caused by specialist drying devices follow. Therefore, it is of great necessity and importance to solve above-mentioned issues and simultaneously endow the aerogels with emergent features and functionalities.

Dynamic covalent polymer networks (DCPNs) $(12,13)$, which possess the benefits of thermosets yet retain re-processability resembling thermoplastics, have been widely explored in synthetic chemistry and materials science (14-21). Under certain conditions, the dynamic networks keep stable, but polymer chains could be depolymerized or rearrangement of the topological network 
structures could occur once dynamic covalent bonds are activated (22-25). When it comes to constructing aerogels, dynamic covalent polymer networks would be a great candidate in consideration of the following merits: (i) The dynamic nature of DCPNs would endow the aerogels with repairability and chemical recyclability, which are scarcely attainable by traditional aerogels (e.g., inorganic silica aerogels and organic resorcinol-formaldehyde aerogels). (ii) The reversible crosslinked DCPNs are able to generate robust skeletons that would prevent gel shrinkage and/or cracking during the drying process and synchronously ensure mechanical performance of the resultant aerogels. (iii) It would greatly enrich the family of aerogels due to a variety of types of DCPNs. As such, we envision that the DCPNs are able to promote the development of aerogels to the next level of simplicity, practicality, and sustainability. Nevertheless, dynamic covalent polymer chemistry has been remained unexploited to fabricate monolithic aerogels.

Herein, we present a straightforward one-pot, mild, and catalyst-free polycondensation strategy via dynamic imine chemistry along with the combination of the low-cost and promising ambient pressure drying to construct monolithic dynamic covalent polymer aerogels (DCPAs). In specific, we first synthesized a polyimine oligomer using terephthalaldehyde (TA) and diethylenetriamine (DETA). Subsequently, tris(2-aminoethyl)amine (TREN) as a crosslinker was applied to induce the formation of highly cross-linked but dynamic polyimine gels. After solvent exchanges and ambient pressure drying, the resulting DCPAs showed low shrinkage that was comparable to those of the aerogels prepared by supercritical drying and freeze drying. Benefiting from the reversible dynamic covalent bonds as well as the robust gel skeletons, our DCPAs simultaneously exhibit flexibility, weldability, and repairability. Moreover, the DCPAs could be depolymerized into soluble oligomers and/or monomers readily by introducing an excess of free amine groups. Impressively, new monolithic aerogels were able to be regenerated from the recyclable solution after the free amine groups were consumed, thereby achieving a closed-loop chemical recyclability. Finally, we exploited the porous structures and robust skeletons to demonstrate the multifunctionality of our DCPAs, such as thermal insulation and emulsion separation.

\section{Results \\ Design, Fabrication, Structural Characterization, and Basic Properties of The DCPAs.}

To better fabricate DCPAs, we abide by three criteria: (i) The preparation process should be simple and suitable for large-scale production. (ii) The light DCPAs should possess qualified mechanical properties to enhance the practicability. (iii) The skeleton of DCPAs should contain abundant dynamic bonds to realize chemical recyclability. To meet the first requirement, the ambient pressure drying that avoids the use of special equipments such as supercritical dryer and freeze dryer, is adopted. Polyimine, prepared from commercially available dialdehyde, diamine, and triamine with readily controllable polycondensation process, represents an ideal candidate. On one hand, the high crosslinking density can ensure the mechanical properties in the second criterion. On the other hand, plenty of reversible imine bonds are able to satisfy the last requirement.

The chemical structures of compounds used in this study and fabrication process of DCPAs are illustrated in Fig. 1, A and B. TA and DETA were firstly chosen to synthesize a polyimine oligomer (Fig. 1B). After prepolymerization, TREN as a crosslinker was added (Fig. 1B). Due to the high reactivity between aldehyde and amine groups, the sol-gel transition was observed prior to macroscopic phase separation (Fig. 1B). After solvent exchanges and ambient pressure drying, we obtained DCPAs with good appearance. On one hand, addition of the TREN into the oligomer solution led to the formation of 3D cross-linked networks as a gel skeleton that could withstand capillary force during the drying process. On the other hand, abundant imine bonds in the gel skeleton are able to undergo dynamic and reversible transiminination reactions, thereby empowering the possibility of weldability, repairability, and recyclability of the DCPAs. Under the constant 1:0.3:0.47 stoichiometry but variable concentrations of TA, DETA, and TREN, we 
constructed monolithic DCPA-1, -2, and -3, respectively (table S1).

Taking DCPA-3 as an example, in the Fourier-transform infrared (FT-IR) spectrum, the characteristic peak of $\mathrm{C}=\mathrm{N}$ stretch at $1640 \mathrm{~cm}^{-1}$ was prominent but the peak of $\mathrm{C}=\mathrm{O}$ stretch at $1690 \mathrm{~cm}^{-1}$ became very weak, suggestive of the formation of imine bonds (fig. S1). The lightweight DCPA-3 $\left(119 \mathrm{mg} / \mathrm{cm}^{3}\right)$ is able to rest on a fresh gypsophila bud (Fig. 1C). It can also support over 3,300 times of its own weight $(300 \mathrm{mg}, 17.8$ (D) $\times 10.0 \mathrm{~mm}(\mathrm{H}))$ without obvious deformation, showing resistance capacity to compression (Fig. 1D). Due to the moderate reaction condition for imine chemistry, great versatility in controlling the shapes of the DCPAs becomes possible. The monolithic DCPA-3 with different shapes, such as the models of house, duck, horse, and different letters, were readily prepared (Fig. 1E). Intriguingly, the desired shape, like a butterfly, was readily made by cutting with a scissor without any fractures (Fig. 1F), which reflects the processability of our DCPAs. Notably, most aerogels prepared by the sol-gel method are brittle and unmachinable $(26,27)$. A piece of DCPA-3 $(50 \mathrm{mg}, 23(\mathrm{~L}) \times 7.0(\mathrm{~W}) \times 2.5 \mathrm{~mm}(\mathrm{H}))$ can sustain a load of $100 \mathrm{~g}$ without cracking, showing good tensile strength (Fig. $1 \mathrm{G})$. In addition, the DCPA-3 displayed great flexibility (Fig. 1H). It not only could be folded in half, but also could be knotted. Interestingly, the DCPA-3 can rebound without cracking after being released from $25 \mathrm{~cm}$ height and the rebound height was able to reach $\sim 9.0 \mathrm{~cm}$ (Fig. 1I). These demonstrations are helpful to break down the stereotype on the lack of outstanding mechanical performance of aerogels.

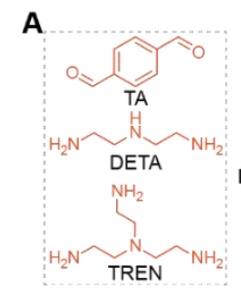

c

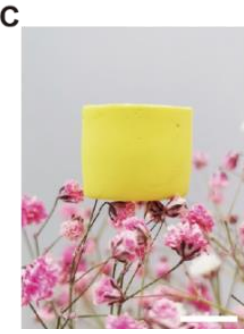

B

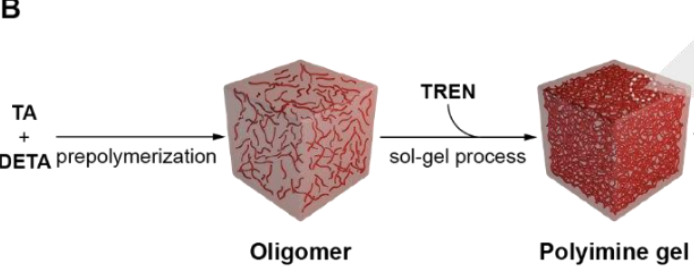

D

$\mathrm{F}$

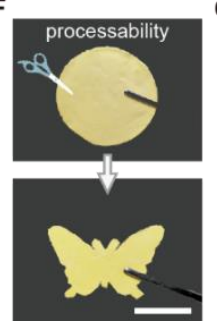

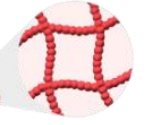

ambient pressure drying

G

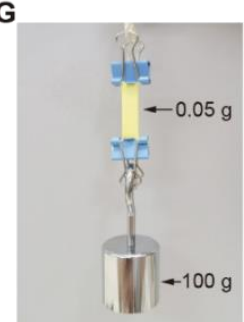

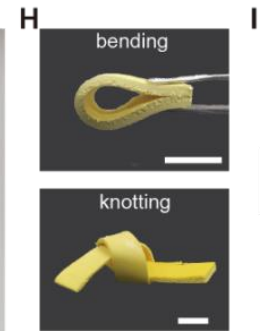

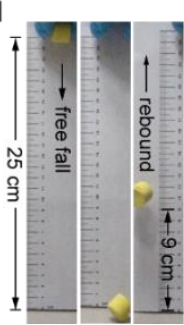

Fig. 1. Illustration of preparation and performances of DCPAs. (A) Chemical structures of compounds used in this study. (B) Schematic illustration of the formation of DCPAs via ambient pressure drying. (C) Photograph showing DCPA-3 resting on a gypsophila. (D) Photograph demonstrating strengthen of DCPA-3 by supporting over 3,300 times of its own weight. (E) Photograph of various shapes made from DCPA-3. (F) Processability of DCPA-3 shown by cutting it into a butterfly with a scissor. (G) Photograph of a piece of DCPA-3 holding a $100 \mathrm{~g}$ load. (H) Photographs showing flexibility of DCPA-3 by bending and knotting tests. (I) Digital images showing rebound elasticity of DCPA-3 by a free-falling process. Scale bars are $1 \mathrm{~cm}(\mathbf{C}, \mathbf{H})$, $5 \mathrm{~cm}(\mathbf{E})$, and $4 \mathrm{~cm}(\mathbf{F})$, respectively.

The basic properties of the DCPAs including bulk density, porosity, and shrinkage, were summarized in table S1. The densities of the DCPAs ranged from $112.5 \pm 2.6$ to $121.4 \pm 11.2$ $\mathrm{mg} / \mathrm{cm}^{3}$. The skeleton density of the polyimine was measured to be $1.30 \mathrm{~g} / \mathrm{cm}^{3}$ by a densimeter, resulting in high porosities of DCPAs in the range of 90.7 to $91.3 \%$. The shrinkage is evaluated by the linear shrinkage ratio (LSR). The DCPA-1 showed the most severe shrinkage (LSR $=22.8 \pm$ $1.5 \%$ ). It could be ascribed to the relatively weak gel skeleton caused by low reactant concentration that failed to withstand the capillary force during the drying process. With the increase of the reactant concentration, the LSR of the DCPAs reduced accordingly. For example, 
the DCPA-3 showed the lowest LSR of $\sim 10.5 \pm 2.8 \%$ that was as good as those of aerogels (fig. S2) prepared by supercritical drying and freeze drying in which the capillary force has been eliminated largely (28). It is worth noting that no special treatments such as hydrophobic modification and high-pressure or vacuum conditions were applied for our ambient-dried DCPAs to weaken the capillary force during the drying process (11). It means that our DCPAs have a great potential to be fabricated in a large-scale without any limitations of technology and devices.

\section{Morphologies and Pore Structures of the DCPAs.}

The morphologies and specific surface areas of the DCPAs were investigated by scanning electron microscopy (SEM), transmission electron microscopy (TEM), and Brunauer-Emmett-Teller measurement (BET), respectively. As shown in the SEM images, all of the DCPA-1 (Fig. 2, A and B), DCPA-2 (Fig. 2, C and D), and DCPA-3 (Fig. 2, E and F) displayed bi-continuous configurations consisting of polyimine gel skeletons and interconnected pores. On one hand, the skeleton was constructed by the interfused micro-nanostructured particles with well dispersed sizes ranging from 400 to $800 \mathrm{~nm}$ (fig. S3), indicative of typical pearl necklace-like aerogel structures. On the other hand, the stacked micro-nanostructured particles formed the interconnected pores ranging from hundred nanometers to a few microns (Fig. 2, A-F). These results were further demonstrated by their TEM images (Fig. 2, G-I). In the BET measurements, we observed that all of the nitrogen adsorption-desorption curves of the DCPAs showed a type III isotherm (fig. S4). The specific surface areas ( $\mathrm{S}_{\mathrm{BET}}$ ) were measured to be 11.9 $\mathrm{m}^{2} / \mathrm{g}$ for DCPA-1, $11.7 \mathrm{~m}^{2} / \mathrm{g}$ for DCPA-2, and $13.5 \mathrm{~m}^{2} / \mathrm{g}$ for DCPA-3 (table $\mathrm{S} 1$ ), which are similar to those of reported aerogels with similar morphologies (29).
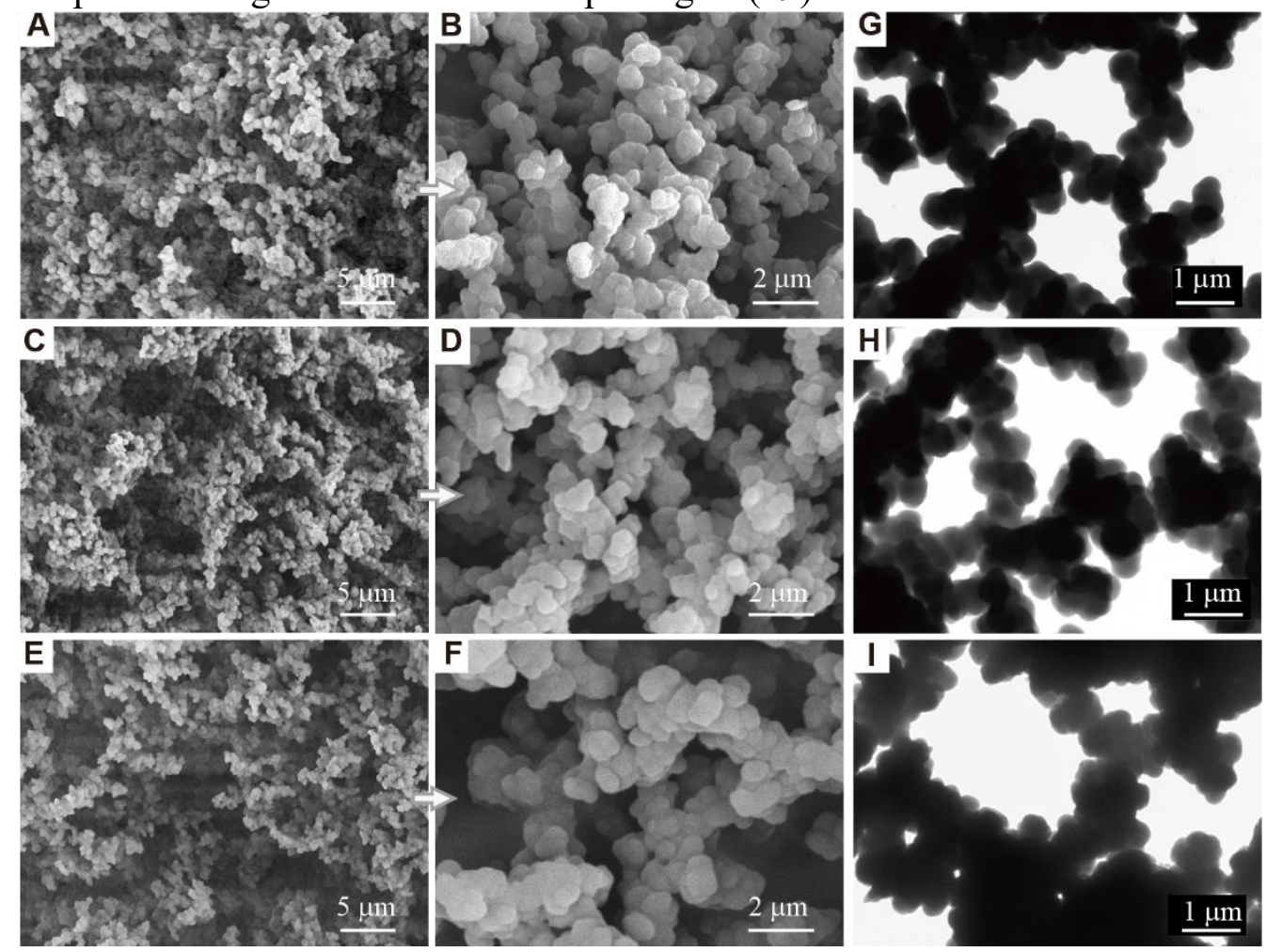

Fig. 2. Structural characterization of the DCPAs. SEM images of DCPA-1 (A, B), DCPA-2 (C, D), and DCPA-3 (E, F). TEM images of DCPA-1 (G), DCPA-2 (H), and DCPA-3 (I).

\section{Fundamental Mechanical Properties of the DCPAs.}

Aerogels oftentimes show a trade-off between low density and high mechanical performance. To explore whether our DCPAs are capable of circumventing this inherent drawback, a series of compressive and three-point bending tests were performed. In sharp contrast to brittle nature of aerogels whose morphological characters are similar to ours $(30,31)$, for example, the mundane 
yet promising resorcinol-formaldehyde aerogels that opened the door to organic aerogels (26), our DCPAs exhibited unexpected mechanical performance including compressibility and flexibility.

The compressive stress-strain curves of the DCPAs at a strain of $80 \%$ were plotted in Fig. $3 \mathrm{~A}$. All of the DCPAs not only tolerated a high compressive strain without any cracks (fig. S5), but also exhibited a nearly $10 \%$ recovery capacity after the pressure was released. Two characteristic deformation regimes, similar to other aerogels (32), were observed in the curves: an elastic regime with a linearly increased stress $(<50 \%$ strain $)$ and a densification regime with an exponentially increased stress $(>50 \%$ strain).

According to the compressive curves of DCPAs at a strain of $80 \%$, their maximum stress and Young's modulus were obtained (Fig. 3B). The DCPA-2 possessed the greatest maximum stress of 2.92 MPa and the values of DCPA-1 and DCPA-3 were 0.98 and $1.80 \mathrm{MPa}$, respectively. It was consistent with the densities of these DCPAs (table S1). Even so, the Young's moduli of DCPA-2 $(0.287 \mathrm{MPa})$ and DCPA-3 $(0.267 \mathrm{MPa})$ are quite similar, which might be due to similar gel skeletons. Subsequently, the specific modulus defined as the ratio of the Young's modulus to the density was calculated (Fig. 3C), which represents a significant parameter for light materials. The specific moduli of DCPA-2 and DCPA-3 were 2.30 and $2.45 \mathrm{kN} \cdot \mathrm{m} / \mathrm{Kg}$, respectively, demonstrating that the DCPAs possess a good anti-compression capacity (Fig. 1D and fig. S6). Moreover, we calculated the energy dissipation of the DCAPs based on the area of the hysteresis loop and the values are $0.11 \mathrm{MJ} / \mathrm{m}^{3}$ for DCPA-1, $0.25 \mathrm{MJ} / \mathrm{m}^{3}$ for DCPA-2, and $0.19 \mathrm{MJ} / \mathrm{m}^{3}$ for DCPA-3 (Fig. 3C). It reveals that our DCPAs hold a promising potential for cushioning materials (9). In consideration of the DCPA-3 with more balanced properties, it would be focused on in the following tests to further investigate the mechanical properties of the DCPAs.

The compression tests of DCPA-3 at different strains of 5, 10, 20,30, and 50\% were carried out (Fig. 3D). When the strain was less than $20 \%$, the curves almost overlapped, indicating that DCPA-3 had decent elasticity in a certain range (Fig. 3D). Upon increasing the strain to $50 \%$, the DCPA-3 still showed considerable recovery capacity. Similar compressive stress-strain curves were also observed on DCPA-1 and DCPA-2 (fig. S7). Afterwards, the compression fatigue test of DCPA-3 was carried out with five loading-unloading cycles at a $30 \%$ strain without an interval (Fig. 3E). The DCPA-3 could retain $95 \%$ of the original maximum stress and the maximum volume deformation was $9.2 \%$ (Fig. 3E and fig. S8), suggestive of a certain degree of fatigue resistance. Dynamic compressive test was further performed on DCPA-3 by a dynamic mechanical analysis (DMA). The storage modulus kept almost stable upon four orders of magnitude from 0.01 to $100 \mathrm{~Hz}$ and the loss modulus only varied by an order of magnitude (Fig. $3 \mathrm{~F}$ ), indicating that elastic response existed in the DCPAs.

The three-point bending curves of diametral deflection as a function of load force were measured to showcase the flexibility of our DCPAs (Fig. 3G). After a large diametral deflection of $30 \mathrm{~mm}$ with a fixture span of $25 \mathrm{~mm}$, all of the DCPAs nearly recovered to their original states without any cracks (Fig. $3 \mathrm{H}$ and fig. S9). The bending flexibility of our DCPAs reached to the level of the reported super-flexible aerogels (33). Notably, the flexibility in compression and bending with large strains have rarely been realized in aerogels prepared by the sol-gel method (33). The mechanical performance of our DCPAs might originate from two key aspects (Fig. 3I): (i) Unlike the traditional silica aerogels with rigid $\mathrm{Si}-\mathrm{O}-\mathrm{Si}$ networks and classic resorcinol-formaldehyde aerogels with short aliphatic hydrocarbon chains $(34,35)$, our DCPAs contain relatively flexible chains in the networks (33). (ii) The interfused micro-nanostructured polyimine particles are able to evolve into fibriform gel skeletons that would promote the recovery after compression and bending tests. 

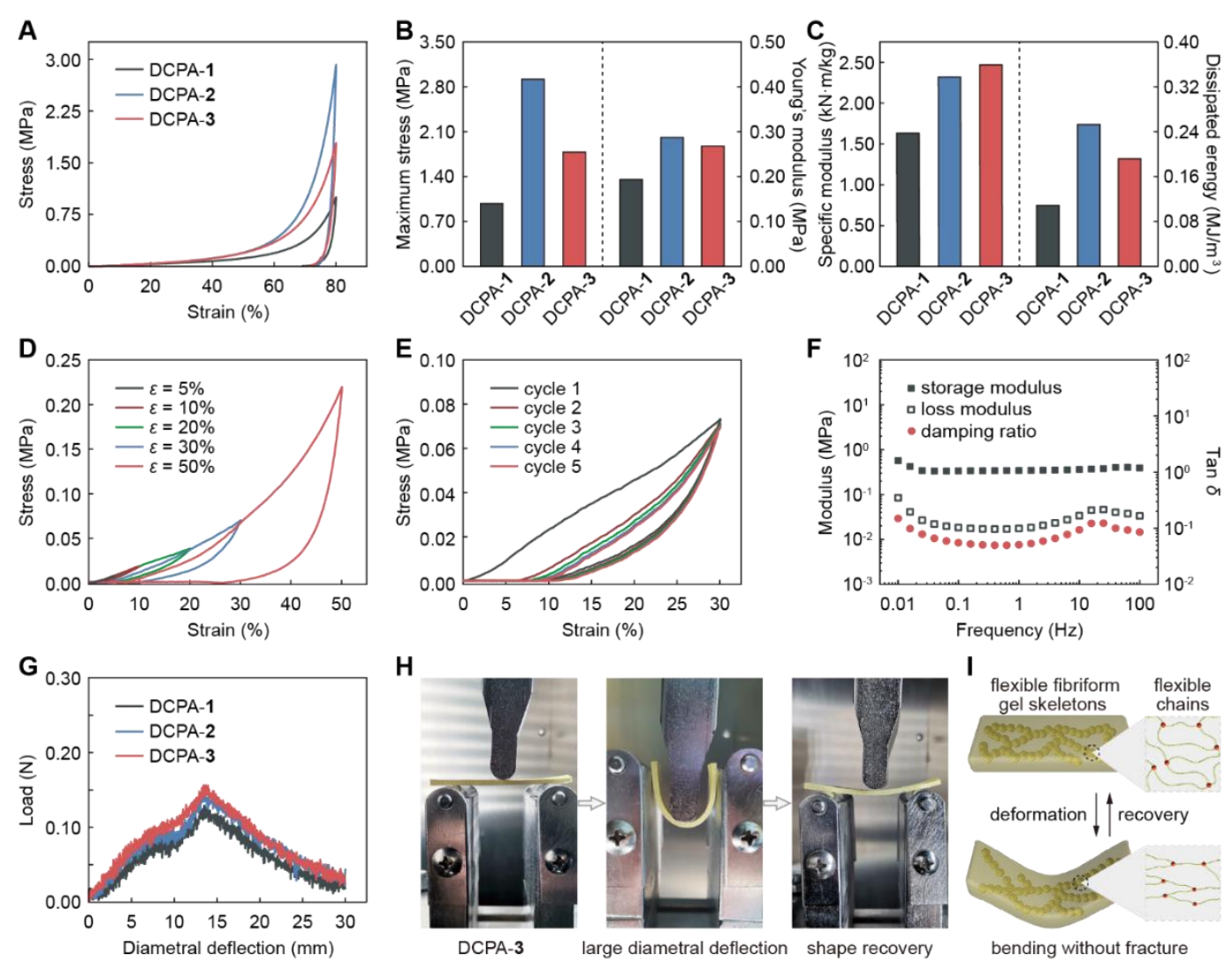

Fig. 3. Mechanical properties of the DCPAs. (A) Compressive stress-strain curves of DCPAs at a strain of $80 \%$ with a deformation rate of $5.0 \mathrm{~mm} / \mathrm{min}$. (B) Maximum stress and Young's moduli of DCPAs. (C) Specific moduli and dissipated energy of DCPAs. (D) Compressive stress-strain curves of DCPA-3 at different strains of 5, 10, 20, 30, and 50\%. (E) Cyclic compressive curves of DCPA-3 at a strain of 30\%. (F) Dynamic compressive behavior of DCPA-3 with oscillatory $\varepsilon$ of $5 \%$ from 0.01 to $100 \mathrm{~Hz}$. (G) Three-point bending curves of DCPAs and corresponding bending images of DCPA-3 with a deformation rate of $1.0 \mathrm{~mm} / \mathrm{min}$ (H). (I) Schematic representation of bending induced deformation of the aerogel skeleton and corresponding polyimine networks.

\section{Weldability, Repairability, and Recyclability of the DCPAs.}

In consideration of abundant dynamic bonds in our DCPAs, we are expecting that there are emerging features on our aerogels that are hardly possessed by traditional aerogels. As a first trial, we tested the weldability of our DCPAs. By applying a small amount of fresh polyimine sol at the cut DCPA-3 followed by ambient pressure drying, the cut aerogel chips were welded (Fig. 4A). In the welded area, we barely observed the fracture surface from both the top and side views (Fig. 4A). To prove the effect, tensile behaviors of DCPA-3 before and after welding were studied (Fig. 4B). Compared with the original tensile curve of DCPA-3, the welded specimen showed good recovery efficiencies in terms of different parameters, including fracture stress (97\%) (fig. S10), strain at break (60\%) (fig. S11), and elastic modulus (80\%) (fig. S12). Furthermore, SEM image showed that the welded surface also formed similar morphologies as observed on DCPA-3 (Fig. 4C), thus facilitating the fusion between the fragmented aerogels (18). It can be explained that the fresh polyimine grew across the fracture sites of the DCPA-3 via the dynamic imine bonds so as to promote the welding effect (36). Along this line, we further demonstrated the repairability of our DCPA-3 (fig. S13). After repaired using polyimine solution, the scratches on the surface of the aerogel film disappeared (fig. S13A). Even in the SEM image, there were no obvious cracks or scratches observed (fig. S13B).

Subsequently, we explored the recyclability of our DCPA-3 by adding an excess of free amine groups to disrupt the stoichiometric balance between aldehyde and amine groups and induce transimination reactions, which could decrease the molecular weight and solubilize the polymer network. In specific, a certain amount of diamine and/or triamine monomers in DMSO as 
recycling solution (see the Supplementary Information for details) was mixed with the debris of DCPA-3 (Fig. 4D). After heating and ultrasonic treatments, the DCPA degraded into soluble oligomers/monomers, which could regenerate polyimine gel upon the addition of dialdehyde monomer in proportion (Fig. 4D). And then, the recycled DCPA was obtained via ambient pressure drying. The LSR of the recycled DCPA was only $15.9 \pm 1.4 \%$ (table S1), representing a satisfying result. The morphology of the recycled DCPA kept the same pearl necklace-like structure formed by the interfused micro-nanostructured particles (figs. S2 and S14). The mechanical properties of the recycled DCPA were also explored. The compressive strain-stress curve of the recycled DCPA was almost overlapped with that of the original DCPA-3 (Fig. 4E). Compared with DCPA-3, the maximum stress (fig. S15) and compression modulus (fig. S16) of the recycled DCPA recovered up to $92.3 \%$ and $73.3 \%$, respectively. The three-point bending curve further proved that the recycled DCPA maintained desirable mechanical performance (Fig. 4F and G). These emerging features, such as weldability, repairability, and recyclability, embody potential values for sustainable development, which would open a novel research perspective for both aerogels and dynamic covalent polymers.
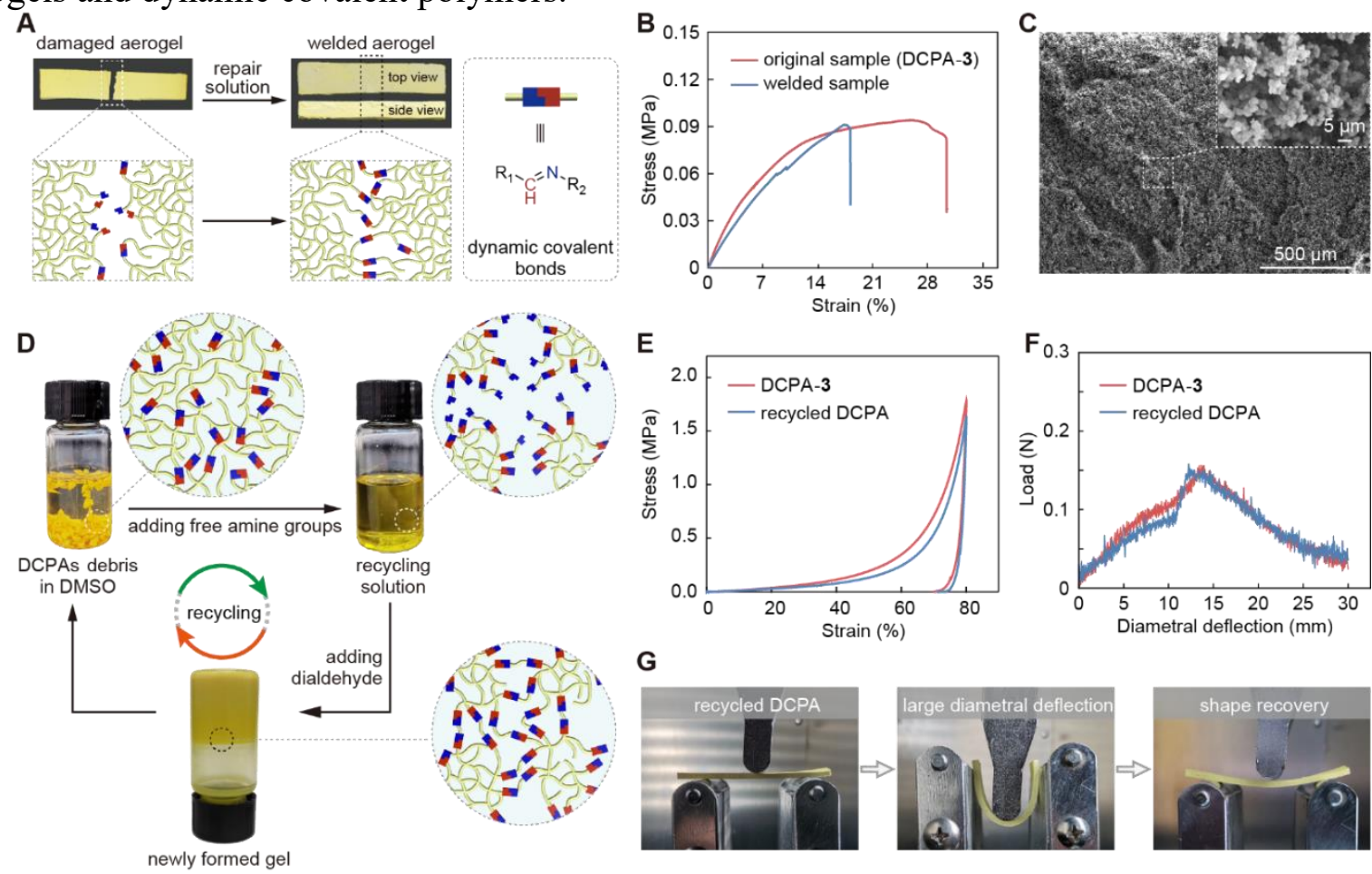

$E$
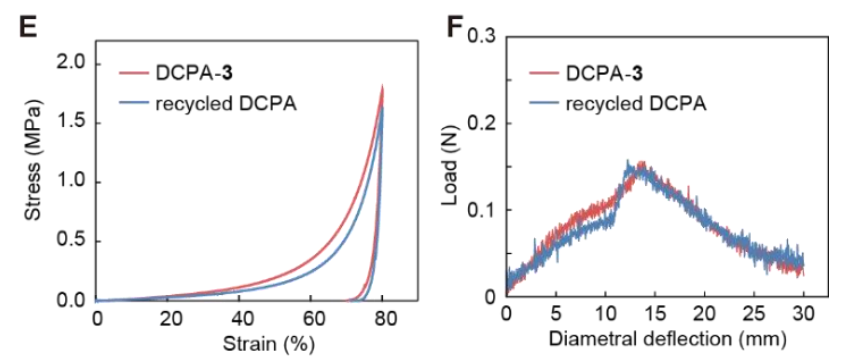

6
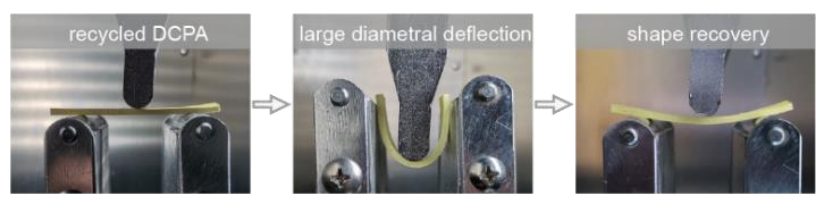

Fig. 4. Weldability, repairability, and recyclability of the DCPAs. (A) Photographs and schematic representation of the welding process of DCPA-3. (B) Tensile stress-strain curves of the virgin and welded samples with a deformation rate of $5.0 \mathrm{~mm} / \mathrm{min}$. (C) SEM images of the fracture surface of the welded sample. (D) Schematic representation of the recyclable process of DCPA-3. (E) Compressive stress-strain curves of DCPA-3 and the recycled DCPA at a strain of $80 \%$ with a deformation rate of $5.0 \mathrm{~mm} / \mathrm{min}$. Three-point bending curves of DCPA-3 and the recycled DCPA with a deformation rate of $1.0 \mathrm{~mm} / \mathrm{min}(\mathbf{F})$ and corresponding bending photographs $(\mathbf{G})$.

\section{Multifunctionality of the DCPAs.}

In the preceding sections, we focused on the basic properties, mechanical properties, and emerging features including weldability, repairability, and closed-loop recyclability of our DCPAs. In this section, we will explore the multifunctionality of the DCPAs such as thermal insulation and oil water separation by taking advantage of their inherent porous structures and robust skeletons.

First of all, we measured the thermal conductivities of DCPA-1, DCPA-2, and DCPA-3 to be $41.6 \pm 2.3,41.8 \pm 1.0$, and $40.9 \pm 1.0 \mathrm{~mW} /(\mathrm{m} \cdot \mathrm{K})$, respectively (Fig. $5 \mathrm{~A}$ ). These values are 
comparable or even superior to those of most aerogels (Figure 5B). The total thermal conductivity $\left(\lambda_{\text {total }}\right)$ of the aerogel is the arithmetic sum of the radiative heat transfer coefficient $\left(\lambda_{r}\right)$, solid thermal conductivity $\left(\lambda_{s}\right)$, and gas thermal conductivity $\left(\lambda_{g}\right)$, which reflects the real thermal transfer coefficient $(37,38)$. The low densities would lead to low $\lambda_{s}$ and the small pore size would result in the decrease of $\lambda_{g}$, which jointly accounted for the low thermal conductivities of our DCPAs (see the Supplementary Information for detailed discussion).

Given the good performance in thermal conductivity, we proceeded to probe the real thermal insulation of the DCPA-3 by an infrared camera and compared it with other common thermal insulating materials including commercial cotton and melamine sponge with the same thickness $(5.0 \mathrm{~mm})$. The measured samples were placed on a $75{ }^{\circ} \mathrm{C}$ heating stage. As shown in the time-temperature curves and the thermal infrared images, the far-end surface temperatures of the commercial thermal insulating cotton and melamine sponge rose quickly within $25 \mathrm{~s}$ and the thermal equilibrium time was less than $50 \mathrm{~s}$ (Fig. 5, C and E). On the contrary, the far-end surface temperature of our DCPA-3 went up more slowly and the thermal equilibrium time extended to around $144 \mathrm{~s}$ with a lower equilibrium temperature (Fig. 5C). Furthermore, the samples were placed on a cold stage $\left(<-50{ }^{\circ} \mathrm{C}\right)$ to study the thermal insulation at low-temperature environment (Fig. 5, D and F). The far-end surface temperature of the melamine sponge decreased rapidly to $-25{ }^{\circ} \mathrm{C}$ within $8 \mathrm{~s}$. For our DCPA-3, it took longer time $(85 \mathrm{~s})$ to reach to the lowest temperature of only $-13.5^{\circ} \mathrm{C}$. The thermal insulation performance both in high and low temperatures for our DCPAs was consistent with their low thermal conductivities.

A

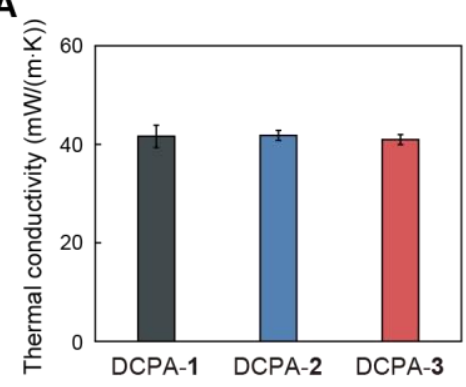

C

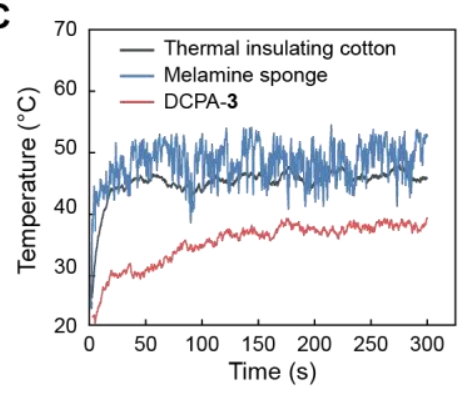

E

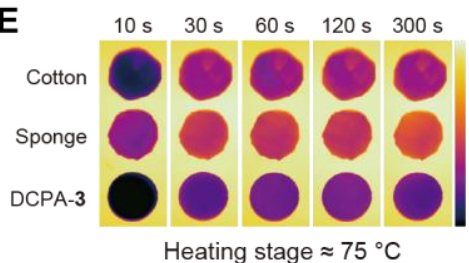

B

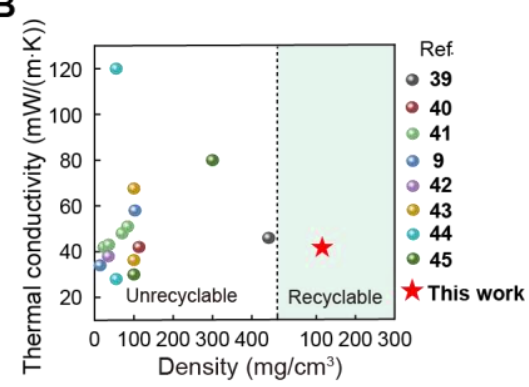

D

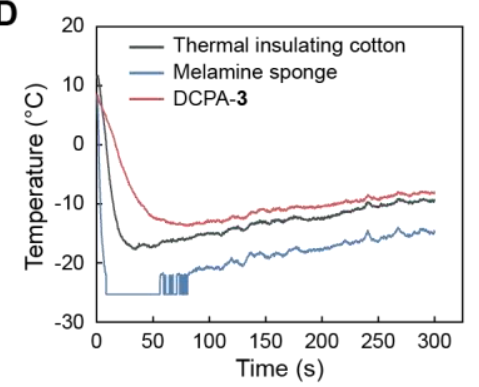

$\mathbf{F}$

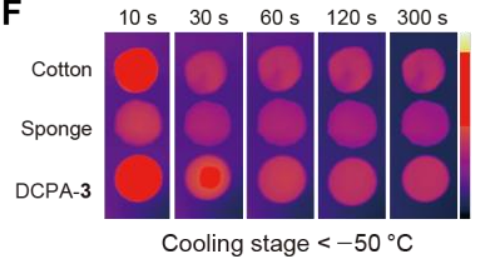

Fig. 5. Thermal insulation of the DCPAs. (A) Thermal conductivities of DCPAs measured at room temperature by the transient plane source method. (B) Ashby plot of thermal conductivity and density of DCPAs and other reported aerogels, including PMSQ aerogel (39), PVA aerogel (40), PU/alumina aerogel (41), MXene/PI aerogel (9), 3D ordered nanofiber aerogel (42), Polymeric woods (43), CNF aerogel (44), and Polyamide aerogel (45). (C) Temperature variation curves of backside of the commercial thermal insulting cotton, melamine sponge with high porosity, and DCPA-3 on a $75^{\circ} \mathrm{C}$ stage for $300 \mathrm{~s}$. (D) Temperature variation curves of backside of commercial thermal insulting cotton, melamine sponge with high porosity, and DCPA-3 on a cold stage $\left(<-50{ }^{\circ} \mathrm{C}\right)$ for $300 \mathrm{~s}$. Corresponding infrared images of the three samples on a $75^{\circ} \mathrm{C}$ stage $(\mathbf{E})$ 
and a cold stage $\left(<-50^{\circ} \mathrm{C}\right)(\mathbf{F})$.

Benefiting from plenty of micro-nanostructured particles in the aerogel frameworks, we conjectured that our DCPAs could be modified readily into hydrophobic materials by low surface energy chemicals (40). The modification of the DCPA-3 was achieved by introducing the hydrophobic surface via the condensation of fluoroalkyl silane (FAS 13) on the aerogel skeleton, which was denoted as DCPA-3-F (Fig. 6A). The energy dispersive spectroscopy (EDS) mapping images clearly showed that the $\mathrm{Si}$ and $\mathrm{F}$ elements were well dispersed in the aerogel matrix (fig. S17). Before hydrophobic modification, the water droplet was adsorbed rapidly by the hydrophilic DCPA-3 during the water contact angle (WCA) measurement (Fig. 6B). In sharp contrast, the WCA of the DCPA-3-F could reach up to $134^{\circ}$ (Fig. 6C). More intuitively, the near-spherical water droplets $(0.3 \mathrm{~mL} /$ droplet) were able to be supported by the DCPA-3-F (Fig. 6D) and the DCPA-3-F could keep afloat atop the water (Fig. 6E), which exhibited the hydrophobicity of our modified DCPA. In light of its high porosity and lipophilicity, the DCPA-3-F was then exploited to separate the immiscible oil-water mixtures. As shown in fig. S18, the DCPA-3-F could remove both the light oil on the top of the water ( $n$-hexane dyed with oil red) and heavy oil under the water (dichloromethane dyed with oil red) rapidly. The success stimulated us to try the separation of surfactant-stabilized emulsion, which is more valuable and challengeable for aerogels. As such, the DCPA-3-F was equipped with a peristaltic pump to construct a simple and continuous separation device by the pumping method (Fig. 6F). When the DCPA-3-F was put into a surfactant-stabilized W/O emulsion, the turbid emulsion was successfully purified into clear oil (Fig. 6F and movie S1), which was further confirmed by the corresponding optical images (Fig. 6, G and H). Such a simple and continuous pumping method for separating emulsion implies that our DCPAs have the potential in pollution control and environmental protection fields (47). 

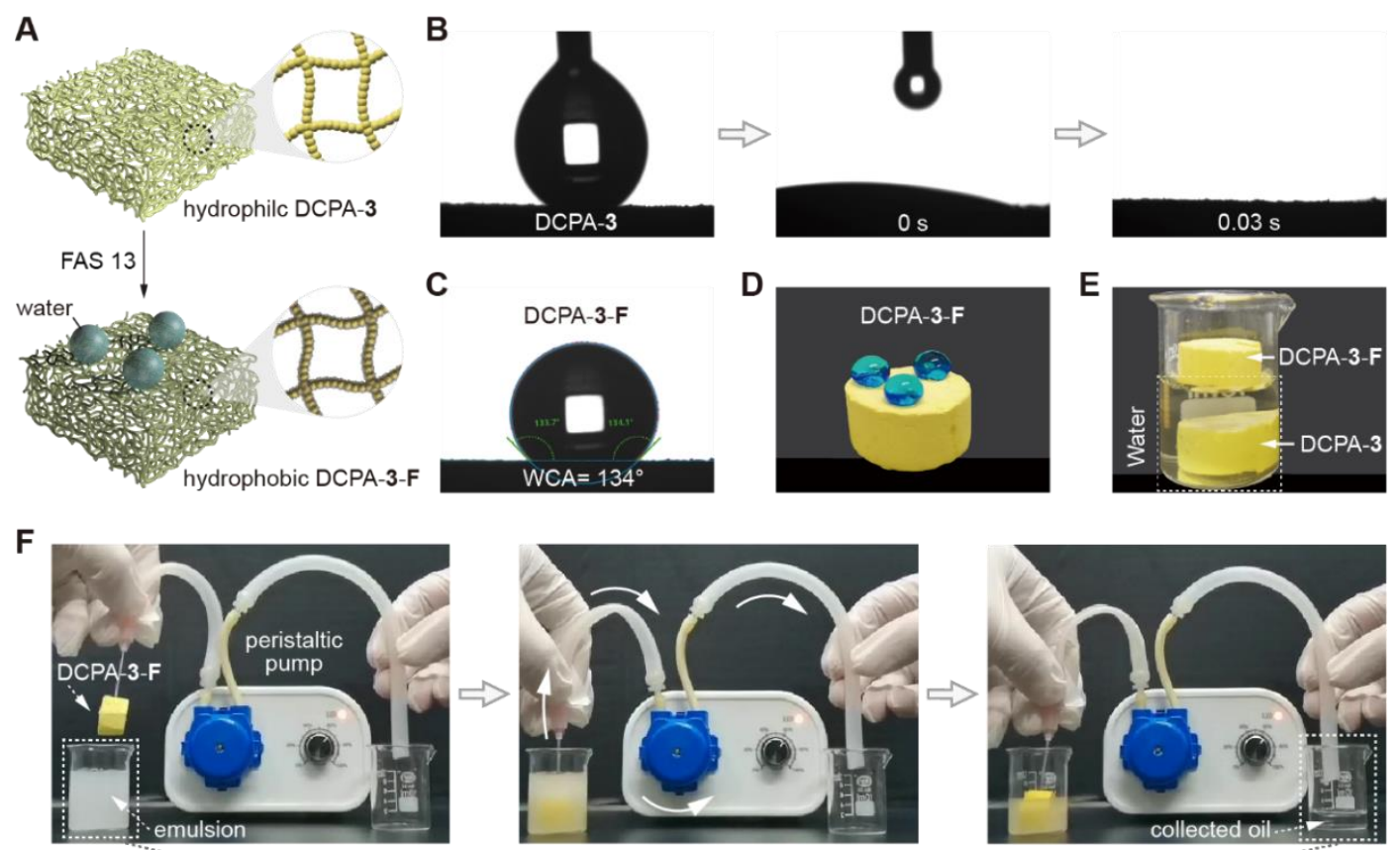

G

H
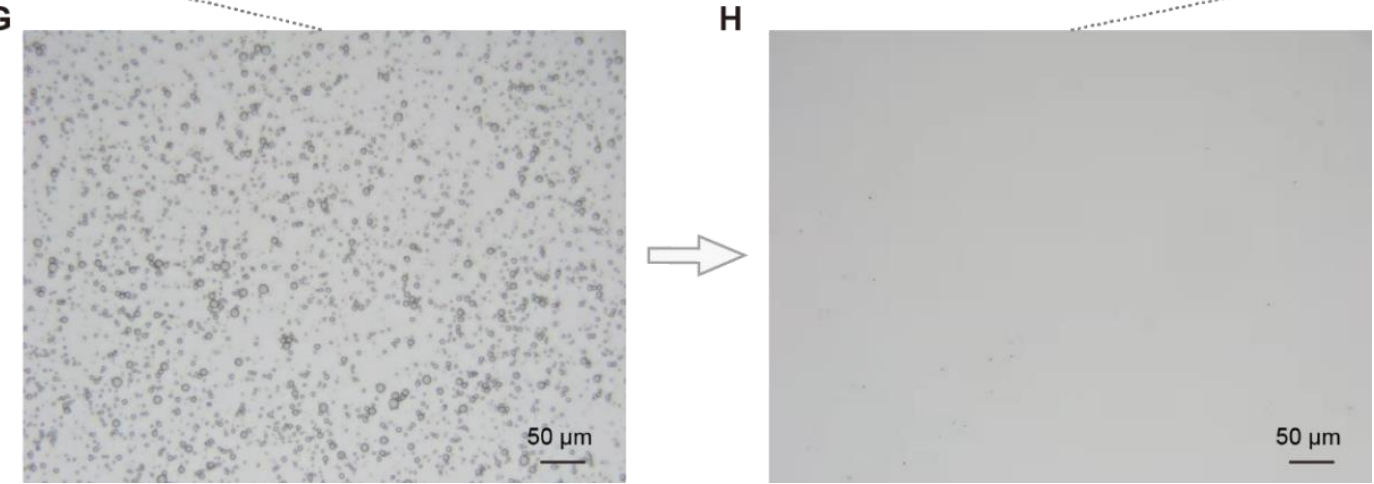

Fig. 6. Application for emulsion separation. (A) Schematic representation of the hydrophobic modification process of DCPA-3. The water contact angle (WCA) measurements of DCPA-3 (B) and DCPA-3-F (C). Photographs of demonstrating the hydrophobicity of DCPA-3-F (D) and the hydrophilicity of DCPA-3 (E). (F) The continuous oil-water emulsion separation apparatus driven by a peristaltic pump. Corresponding optical images before $(\mathbf{G})$ and after separation $(\mathbf{H})$.

\section{Discussion}

In summary, we demonstrate that dynamic covalent polymer networks, an increasingly important research topic, can be employed to fabricate monolithic dynamic covalent polymer aerogels (DCPAs) with newly-developing features such as weldability, repairability, and closed-loop recyclability. The mild and catalyst-free sol-gel process via dynamic imine chemistry and simple ambient pressure drying method make it possible to fabricate DCPAs in large with arbitrary models. Furthermore, our DCPAs not only possessed low density of $\sim 119 \mathrm{mg} / \mathrm{cm}^{3}$ and suppressed linear shrinkage rate of $\sim 10.5 \%$, but also showcased basketball-like rebound ability, large degrees of compression (80\% strain) and bending (diametral deflection with $30 \mathrm{~mm}$ ) without any cracks, and decent tensile strain of $32.7 \%$. In addition, on account of their closed-loop chemical recyclability and robust porous structures, our DCPAs are able to be developed into green, low-cost, and multifunctional materials of thermal insulation and water treatment. This straightforward principle would be suitable for other dynamic covalent bonds to enrich the library of DCPAs. We expect that this work would facilitate the development of both dynamic covalent chemistry and aerogels for encouraging applications as smart and 
environmental-friendly materials.

\section{Supplementary Materials}

Supplementary material for this article is available.

\section{References}

1. I. Gouzman, E. Grossman, R. Verker, N. Atar, A. Bolker, N. Eliaz, Advances in polyimide-based materials for space applications. Adv. Mater. 31, 1807738 (2019).

2. S. S. Kistler, Coherent expanded-aerogels. J. Phys. Chem. 36, $52-64$ (1931).

3. L. Su, H. Wang, M. Niu, S. Dai, Z. Cai, B. Yang, H. Huyan, X. Pan, Anisotropic and hierarchical $\mathrm{SiC} @ \mathrm{SiO}_{2}$ nanowire aerogel with exceptional stiffness and stability for thermal superinsulation. Sci. Adv. 6, eaay6689 (2020).

4. X. Xu, Q. Zhang, M. Hao, Y. Hu, Z. Lin, L. Peng, T. Wang, X. Ren, C. Wang, Z. Zhao, C. Wan, H. Fei, L. Wang, J. Zhu, H. Sun, W. Chen, T. Du, B. Deng, G. J. Cheng, I. Shakir, C. Dames, T. S. Fisher, X. Zhang, H. Li, Y. Huang, X. Duan, Double-negative-index ceramic aerogels for thermal superinsulation. Science 363, 723-727 (2019).

5. D. Lin, P. Y. Yuen, Y. Liu, W. Liu, N. Liu, R. H. Dauskardt, Y. Cui, A silica-aerogel-reinforced composite polymer electrolyte with high ionic conductivity and high modulus. Adv. Mater. 30, 1802661 (2018).

6. G. Zu, K. Kazuyoshi, M. Ayaka, K. Hironori, N. Kazuki, Superflexible multifunctional polyvinylpolydimethylsiloxane-based aerogels as efficient absorbents, thermal superinsulators, and strain sensors. Angew. Chem., Int. Ed. 57, 9722-9727 (2018).

7. X. Cao, J. Zhang, S. Chen, R. J. Varley, K. Pan, 1D/2D nanomaterials synergistic, compressible, and response rapidly 3D graphene aerogel for piezoresistive sensor. $A d v$. Funct.Mater. 30, 2003618 (2020).

8. Y. Si, J. Yu, X. Tang, J. Ge, B. Ding, Ultralight nanofibre-assembled cellular aerogels with superelasticity and multifunctionality. Nat. Commun. 5, 5802 (2014).

9. J. Liu, H.-B. Zhang, X. Xie, R. Yang, Z. Liu, Y. Liu, Z.-Z. Yu, Multifunctional, superelastic, and lightweight Mxene/polyimide aerogels. Small 14, 1802479 (2018).

10. X. Zhang, W. Li, P. Song, B. You, G. Sun, Double-cross-linking strategy for preparing flexible, robust, and multifunctional polyimide aerogel. Chem. Eng. J. 381, 122784 (2020).

11. S. S. Prakash, C. J. Brinker, A. J. Hurd, S. M. Rao, Silica aerogel films prepared at ambient pressure by using surface derivatization to induce reversible drying shrinkage. Nature 374, 439-443 (1995).

12. N. Zheng, Y. Xu, Q. Zhao, T. Xie, Dynamic covalent polymer networks: a molecular platform for designing functions beyond chemical recycling and self-healing. Chem. Rev. 3, 1716-1745 (2021).

13. W. Zou, B. Jin, Y. Wu, H. Song, Y. Luo, F. Huang, J. Qian, Q. Zhao, T. Xie, Light-triggered topological programmability in a dynamic covalent polymer network. Sci. Adv. 6, eaaz2362 (2020).

14. K. Liu, Y. Jiang, Z. Bao, X. Yan, Skin-inspired electronics enabled by supramolecular polymeric materials. CCS Chem. 1, 431-447 (2019).

15. Z. Zhang, L. Cheng, J. Zhao, L. Wang, K. Liu, W. Yu, X. Yan, Synergistic covalent and supramolecular polymers for mechanically robust but dynamic materials. Angew. Chem., Int. Ed. 59, 12139-12146 (2020).

16. Z. Zhang, L. Cheng, J. Zhao, H. Zhang, X. Zhao, Y. Liu, R. Bai, H. Pan, W. Yu, X. Yan, Muscle-mimetic synergistic covalent and supramolecular polymers: phototriggered 


\section{Q. Xia, C. Chen, Y. Yao, J. Li, S. He, Y. Zhou, T. Li, X. Pan, Y. Yao, L. Hu, A strong, biodegradable and recyclable lignocellulosic bioplastic. Nat. Sustain. (2021). https://doi.org/10.1038/s41893-021-00705-7}

formation leads to mechanical performance boost. J. Am. Chem. Soc. 143, 902-911 (2021).

17. Q. Zhang, C.-Y. Shi, D.-H. Qu, Y.-T. Long, B. L. Feringa, H. Tian, Exploring a naturally tailored small molecule for stretchable, self-healing, and adhesive supramolecular polymers. Sci. Adv. 4, eaat8192 (2018).

18. B. Qin, S. Zhang, P. Sun, B. Tang, Z. Yin, X. Cao, Q. Chen, J.-F. Xu, X. Zhang, Tough and multi-recyclable cross-linked supramolecular polyureas via incorporating noncovalent bonds into main-chains. Adv. Mater. 32, 2000096 (2020).

19. D. Xia, P. Wang, X. Ji, N. M. Khashab, J. L. Sessler, F. Huang, Functional supramolecular polymeric networks: the marriage of covalent polymers and macrocycle-based host-guest interactions. Chem. Rev. 120, 6070-6123 (2020).

21. X. Ji, Z. Li, X. Liu, H.-Q. Peng, F. Song, J. Qi, J. W. Y. Lam, L. Long, J. L. Sessler, B. Z. Tang, A functioning macroscopic "rubik's cube" assembled via controllable dynamic covalent interactions. Adv. Mater. 31, 1902365 (2019).

22. M. E. Belowich, J. F. Stoddart, Dynamic imine chemistry. Chem. Soc. Rev. 41, 2003-2024 (2012).

23. P. Taynton, K. Yu, R. K. Shoemaker, Y. Jin, H. J. Qi, W. Zhang, Heat- or water-driven malleability in a highly recyclable covalent network polymer. Adv. Mater. 26, 3938-3942 (2014).

24. Y. H. Jin, Z. P. Lei, P. Taynton, S. F. Huang, W. Zhang, Malleable and Recyclable Thermosets: The Next Generation of Plastics. Matter 1, 1456-1493 (2019).

25. M. Häußler, M. Eck, D. Rothauer, S. Mecking, Closed-loop recycling of polyethylene-like materials. Nature 590, 423-427 (2021).

26. R. W. Pekala, Organic aerogels from the polycondensation of resorcinol with formaldehyde. J. Mater. Sci. 24, 3221-3227 (1989).

27. N. Zion, J. C. Douglin, D. A. Cullen, P. Zelenay, D. R. Dekel, L. Elbaz, Porphyrin aerogel catalysts for oxygen reduction reaction in anion-exchange membrane fuel cells. Adv. Funct. Mater. 31, 2100963 (2021).

28. J. Yang, W. Yang, W. Chen, X. Tao, An elegant coupling: Freeze-casting and versatile polymer composites. Prog. Polym. Sci. 109, 101289 (2020).

29. G. Hasegawa, T. Shimizu, K. Kanamori, A. Maeno, H. Kaji, K. Nakanishi, Highly flexible hybrid polymer aerogels and xerogels based on resorcinol-formaldehyde with enhanced elastic stiffness and recoverability: insights into the origin of their mechanical properties. Chem. Mater. 29, 2122-2134 (2017).

30. X. Li, J. Wang, Y. Zhao, X. Zhang, Template-free self-assembly of fluorine-free hydrophobic polyimide aerogels with lotus or petal effect. ACS Appl. Mater. Interfaces 10, 16901-16910 (2018).

31. M. A. B. Meador, E. J. Malow, R. Silva, S. Wright, D. Quade, S. L. Vivod, H. Guo, J. Guo, M. Cakmak, Mechanically strong, flexible polyimide aerogels cross-linked with aromatic triamine. ACS Appl. Mater. Interfaces 4, 536-544 (2012).

32. Y. Si, Q. Fu, X. Wang, J. Zhu, J. Yu, G. Sun, B. Ding, Superelastic and superhydrophobic nanofiber-assembled cellular aerogels for effective separation of oil/water emulsions. ACS Nano 9, 3791-3799 (2015).

33. G. Zu, T. Shimizu, K. Kanamori, Y. Zhu, A. Maeno, H. Kaji, J. Shen, K. Nakanishi, Transparent, superflexible doubly cross-linked polyvinylpolymethylsiloxane aerogel superinsulators via ambient pressure drying. ACS Nano 12, 521-532 (2018).

34. S. Zhao, G. Siqueira, S. Drdova, D. Norris, C. Ubert, A. Bonnin, S. Galmarini, M. 
Ganobjak, Z. Pan, S. Brunner, G. Nyström, J. Wang, M. M. Koebel, W. J. Malfait, Additive manufacturing of silica aerogels. Nature 584, 387-392 (2020).

35. Z.-L. Yu, Z.-Y. Wu, S. Xin, C. Qiao, Z.-Y. Yu, H.-P. Cong, S.-H. Yu, General and straightforward synthetic route to phenolic resin gels templated by chitosan networks. Chem. Mater. 26, 6915-6918 (2014).

36. C. Shi, Z. Zou, Z. Lei, P. Zhu, W. Zhang, J. Xiao, Heterogeneous integration of rigid, soft, and liquid materials for self-healable, recyclable, and reconfigurable wearable electronics. Sci. Adv. 6, eabd0202 (2020).

37. X. Lu, M. C. Arduini-schuster, J. Kuhn, O. Nilsson, J. Fricke, R. W. Pekala, Thermal conductivity of monolithic organic aerogels. Science 255, 971-972 (1992).

38. B. Wicklein, A. Kocjan, G. Salazar-Alvarez, F. Carosio, G. Camino, M. Antonietti, L. Bergström, Thermally insulating and fire-retardant lightweight anisotropic foams based on nanocellulose and graphene oxide. Nat. Nanotechnol. 10, 277-283 (2015).

39. G. Hayase, K. Kugimiya, M. Ogawa, Y. Kodera, K. Kanamori, K. Nakanishi, The thermal conductivity of polymethylsilsesquioxane aerogels and xerogels with varied pore sizes for practical application as thermal superinsulators. J. Mater. Chem. A 2, 6525-6531 (2014).

40. O.-J. Lee, K.-H. Lee, T. Jin Yim, S. Young Kim, K.-P. Yoo, Determination of mesopore size of aerogels from thermal conductivity measurements. J. Non-Cryst. Solids 298, 287-292 (2002).

41. H. Xie, W. Yang, A. C. Y. Yuen, C. Xie, J. Xie, H. Lu, G. H. Yeoh, Study on flame retarded flexible polyurethane foam/alumina aerogel composites with improved fire safety. Chem. Eng. J. 311, 310-317 (2017).

42. Y. Kobayashi, T. Saito, A. Isogai, Aerogels with 3D ordered nanofiber skeletons of liquid-crystalline nanocellulose derivatives as tough and transparent insulators. Angew. Chem., Int. Ed. 53, 10394-10397 (2014).

43. Z.-L. Yu, N. Yang, L.-C. Zhou, Z.-Y. Ma, Y.-B. Zhu, Y.-Y. Lu, B. Qin, W.-Y. Xing, T. Ma, S.-C. Li, H.-L. Gao, H.-A. Wu, S.-H. Yu, Bioinspired polymeric woods. Sci.Adv. 4, eaat7223 (2018).

44. J. Song, C. Chen, Z. Yang, Y. Kuang, T. Li, Y. Li, H. Huang, I. Kierzewski, B. Liu, S. He, T. Gao, S. U. Yuruker, A. Gong, B. Yang, L. Hu, Highly compressible, anisotropic aerogel with aligned cellulose nanofibers. ACS Nano 12, 140-147 (2018).

45. J. C. Williams, B. N. Nguyen, L. McCorkle, D. Scheiman, J. S. Griffin, S. A. Steiner, III, M. A. B. Meador, Highly porous, rigid-rod polyamide aerogels with superior mechanical properties and unusually high thermal conductivity. ACS Appl. Mater. Interfaces 9, 1801-1809 (2017).

46. L. Jiang, L. Feng, Bioinspired Intelligent Nanostructured Interfacial Materials (World Scientific ;Chemical Industry Press, 2010), pp. 364.

47. J. Wu, F. Xu, S. Li, P. Ma, X. Zhang, Q. Liu, R. Fu, D. Wu, Porous polymers as multifunctional material platforms toward task-specific applications. Adv. Mater. 31, 1802922 (2019).

\section{Acknowledgments}

We thank our colleagues from the instrumental analysis center of Shanghai Jiao Tong University for support and assistance in the research.

Funding: X.Y. acknowledges the financial support of the NSFC/China (21901161 and 22071152) and Natural Science Foundation of Shanghai (20ZR1429200). 


\section{Data and materials availability:}

All data needed to evaluate the conclusions in the paper are present in the paper and/or the Supplementary Materials. Additional data related to this paper may be requested from the authors.

\section{Supplementary Materials}

